\title{
ORAL CHRONIC GRAFT VERSUS HOST DISEASE IN AN IMMUNOCOMPETENT PATIENT: A CASE REPORT
}

\author{
P. Redwin Dhas Manchil ${ }^{1}$, Eugenia Sherubin², S. Karthiga Kannan ${ }^{3}$
}

\section{HOW TO CITE THIS ARTICLE:}

P. Redwin Dhas Manchil, Eugenia Sherubin, S. Karthiga Kannan. "Oral Chronic Graft versus Host Disease in a Immunocompetent Patient: A Case Report". Journal of Evolution of Medical and Dental Sciences 2014; Vol. 3, Issue 23, June 09; Page: 6521-6525, DOI: $10.14260 /$ jemds/2014/2776

\begin{abstract}
Graft-versus-host disease (GVHD) is a complication which occurs in an individual who has received allogenic transplant. This was first noticed in individuals who had undergone bone marrow transplantation. Since then it has been described in solid organ transplantation and even transfusion of blood and blood products. Transfusion-associated GVHD (TA-GVHD) is acute or chronic in nature. Acute TA-GVHD is rare and usually runs a fulminant and fatal course. In its chronic form, oral cavity is frequently affected with wide variety of signs and symptoms resulting in significant short and long-term complications ranging from mucosal sensitivity and limited oral in take to secondary malignancy and early death. Herewith we report a case of oral cGVHD in an immuno competent patient who underwent transfusion of platelets from an unrelated donor.
\end{abstract}

KEYWORDS: platelet Transfusion, Lichenoid reaction, oral cavity, buccal mucosa and tongue.

INTRODUCTION: Hematopoietic stem cell transplant (HSCT) includes transfusion of whole blood or blood cellular products such as red blood cells, platelets and granulocyte concentrates and even fresh plasma from a related or unrelated volunteer donor matched to the recipient (Allogenic transplant).[1]

For many years this Allogenic transplant is a curative option for many diseases such as hematological malignancies, autoimmune diseases, hemoglobinopathies etc. However once received, as many as half of these allogenic transplant recipients experiences a post-transplant complication known as Graft-versus-host disease (GVHD).[2]

GVHD can be classified as either acute or chronic. ${ }^{[3]}$ Acute manifests mainly as erythema and maculopapular cutaneous lesions, liver dysfunction, oral mucositis, upper and lower gastrointestinal involvement occurring within 100days of HSCT.[4] However Chronic GVHD (cGVHD) is the most common complication following HSCT developing within three years after HSCT.

Although cGVHD is a systemic condition mediated by alloreactive donor-derived lymphocytes, the clinical impact and associated morbidity are typically experienced at the level of the individual target organ, where localized and anatomically distinct immune activity (eg, mucosal immunity) may play a major pathogenic role in mediating disease activity.

The oral cavity is one of the most frequently affected area with wide variety of signs and symptoms resulting in significant short and long-term complications ranging from mucosal sensitivity and limited oral intake to secondary malignancy and early death.[5] The clinical manifestations of Oral cGVHD includes mucosal lesions, Xerostomia, mucoceles and sclerotic fibrosis of oral tissues each of which can contribute to significant morbidity and late complications.[1]

CASE REPORT: A 36 years old male patient with no history of deleterious habits was referred to our department with a chief complaint of grayish white discoloration in the left buccal mucosa which was associated with occasional pain and burning sensation since a year which aggravates and recedes on 
its own. The medical history revealed that the patient was diagnosed with idiopathic thrombocytopenia 2 years back and underwent 5 pints of platelet transfusion once. And he was not under any medications. Intraoral examination revealed a grayish white discoloration in the left posterior buccal mucosa measuring approximately $4 \times 3 \mathrm{cms}$ extending anteriorly from first molar region to third molar region posteriorly above the occlusal plane (fig. 1). The lesion was seen scattered with white papules throughout and surrounded with an erythematous border.

The surface of the lesion was rough, non scrapable and non-tender on palpation. On examination of tongue the right lateral border of the tongue revealed grayish white discoloration with striae scattered all over the lesion (fig. 2). And he didn't have any kind of tooth restorations. So based on the history and clinical examination we considered a provisional diagnosis of Transfusion associated Chronic Graft Versus Host Disease considering Lupus erythematosis as a differential diagnosis. Subsequently the Patient underwent ANA test which helped us ruling out the possibility of Lupus erythematosis.

The serum biochemistry, including liver and renal function tests, was within normal limits. An incisional biopsy was performed on the left buccal mucosa for histopathological examination. The histopathalogist offered a diagnosis of non-specific lichenoid mucositis. Therefore a final diagnosis of Ta-GVHD was rendered based on the staging and scoring of National Institute of Health Consensus Development project on GVHD, history of transfusion, and exclusion of other causes.

The lesion was treated with topical application of $0.1-0.5 \%$ Triamcinalone thrice a day for 2 weeks. On review after 3 weeks the lesion showed complete remission (fig. 3 \& 4). However the patient is undergoing periodic screening and been scheduled under long term follow up.

DISCUSSION: Despite advances in HLA matching and GVHD prophylaxis, cGVHD continues to be a significant complication of allogenic HSCT.

All cellular blood products contain mature T cells, which act as immunocompetent cells in the graft and can mount GVHD. Under normal circumstances, an immunocompetent host destroys the donor cells, thereby preventing them to mount a graft-versus-host response. However, in immunodeficient state, the host cannot reject the foreign $\mathrm{T}$ cells, which proliferates resulting in GVHD.

Rarely TA-GVHD has been described in an immunocompetent host, when the host and the graft share HLA haplotype where the host fails to recognize the transfused donor cells as foreign and cannot reject them. Probably this could be the reason why our patient experienced a transfusion associated cGVHD despite being immunocompetent.[6]

Though cGVHD is a multisystem disorder, large single-center study reports $80 \%$ of the patients demonstrating oral involvement. Oral features are generally present at the time of cGVHD diagnosis and may represent the initial clinical manifestations and oral involvement is probably the most common location of single-site disease. [7]

However the spectrum of clinical presentation in the oral cavity is diverse in its type and severity of tissue changes.[1] Typically, cGVHD oral mucosal lesions are characterized as erythema, lichenoid and ulcerative lesions involving any site including labial mucosa, buccal mucosa, tongue, hard and soft palate, floor of mouth and gingiva.

Erythema manifests as redness without obvious tissue breakdown and Lichenoid lesions are characterized by white or milky reticular streaks or lacey lines resembling Wickham's striae 
observed in oral lichen planus and are often not painful. These oral mucosal lesions are considered diagnostic for oral cGVHD in the context of post-HSCT oral evaluation.[4] Various authors have also reported recurrent mucoceles and Sclerotic fibrosis in these patients however these findings were not observed in our case.

If it is present then Mucoceles will be mucus-filled, painless, superficial blisters developing predominantly on the palate but can also be observed on the labial and buccal mucosa and tongue, or wherever there are minor salivary glands. Sclerotic fibrosis of oral tissue is generally rare but when present it resembles tissue changes observed in patients with scleroderma.

This is often a consequence of chronic inflammation of oral tissues resulting in scarring and restricted mouth movement which in turn may lead to difficulty in eating and poor oral hygiene potentially contributing to secondary infection and malnutrition.[1,5]

Diagnosis of oral cGVHD often requires exclusion of other causes such as Herpes Simplex ${ }^{[5]}$ and candidiasis as these infections often complicate the differential diagnosis of cGVHD. Samples should be taken for any suspected oral infection and analyzed by culturing for yeast or PCR analysis of viral involvement, as appropriate for individual cases.

Drug reactions and recurrent or new malignant lesions must be excluded prior to a definitive diagnosis of oral cGVHD. Further testing in support of oral cGVHD diagnosis includes biopsy of the lesions (non-ulcerated tissue) or adjacent tissue, including labial Minor Salivary Glands in xerostomic patients.[1]

Clinically chronic GVHD can be also staged and scored based on the National Institute of Health (NIH) Consensus Development project on GVHD.[8] Accordingly the present case was categorized as a mild form of Ta-GVHD.

The clinical management of cGVHD with multisystem involvement requires systemic corticosteroids and/or other immunomodulatory agents. However when the condition is limited to oral cavity the mainstay of management is intensive topical corticosteroids with no need of systemic therapy. ${ }^{[9]}$ The efficacy of topical therapies depends on active medication penetrating into the oral mucosa, potency of the agent, the vehicle and formulation in which it is delivered, and the duration/ frequency of application.[10,11]

The ultimate goals of oral cGVHD therapy include reduction of symptoms, resolution of painful lesions, and screening for prevention and management of secondary complications. And all patients should be educated on maintaining good oral hygiene with daily tooth brushing, flossing, regular professional scalings and elective dental visits during the first year after transplantation. ${ }^{[9,12]}$

Given the significantly increased risk of developing oral squamous cell carcinoma, all patients with oral cGVHD require periodic screening for suspicious abnormalities, such as focal masses, atypical plaques, non-healing ulcers and indurations..$^{[5,13]}$

Areas of suspicious changes require incisional biopsy and histopathologic examination. It appears that these secondary oral cancers may be associated with higher rates of recurrence and poorer long-term survival compared with de novo squamous cell carcinoma of the oral cancer in nonHSCT patients.[14]

In conclusion, Oral cGVHD is a frequent and potentially serious complication after allogenic HSCT. Although oral lesions are not life threatening, it is definitely associated with increased risk of developing oral squamous cell carcinoma. This condition often goes unnoticed at the transplant 
centers due to the lack of clinically trained transplant specialists in examining and diagnosing complex oral lesions. Henceforth the oral cavity of all those individuals who had undergone HSCT should be subjected to thorough oral examinations at regular intervals by the oral medicine specialists for optimal management.

\section{REFERENCES:}

1. JW Mays, H Fassil, DA Edwards, SZ Pavletic, CW Bassim (2013) Oral Chronic graft-versus-host disease: Current pathogenesis, therapy, and research. Oral Diseases 19: 3227-346. DOI: $10.1111 /$ odi.12028.

2. Li HW, Sykes M (2012). Emerging concepts in haematopoieticcell transplantation. Nat Rev Immunol 12: 403-416.

3. Goker H, Haznedaroglu IC, Chao NJ (2001). Acute graft-vs-host disease: pathobiology and management. Exp Hematol 29: 259-277.

4. Filipovich AH, Weisdorf D, Pavletic S et al (2005). National Institutes of Health consensus development project on criteria for clinical trials in chronic graft-versus-host disease: I. Diagnosis and staging working group report. Biol Blood Marrow Transplant 11: 945-956.

5. Nathaniel Treister, Christine Duncan, Corey Cutler et al. (2012) How we treat oral chronic graftversus-host disease. Blood120: 3407-3418. doi: 10.1182/blood-2012-05-393389.

6. Anju Gupta, Deepak Bansal, Rashna Dass et al. (2004). Transfusion Associated Graft versus Host Disease - A case report. Indian Pediatrics. 41: 1260-1264.

7. Schubert MM, Correa ME (2008). Oral graft-versus-host disease. Dent Clin North Am 52: 79109, viii-ix.

8. Alexandra H. Filipovich, Daniel Weisdorf, Steven Pavletic, Gerard Socie, John R. Wingard, Stephanie J. Lee, et al. (2005) National Institutes of Health Consensus Development Project on Criteria for Clinical Trials in Chronic Graft-versus-Host Disease: I. Diagnosis and Staging Working Group Report. Biology of Blood and Marrow Transplantation 11:945-955 (2005). doi: 10.1016/j.bbmt.2005.09.004.

9. Couriel D, Carpenter PA, Cutler C, et al. (2006) Ancillary therapy and supportive care of chronic graft versus-host disease: National Institutes of Health Consensus Development Project on Criteria for Clinical Trials in Chronic Graft-Versus-Host Disease: V. Ancillary Therapy and Supportive Care Working Group Report. Biol Blood Marrow Transplant. 2006; 12 (4): 375-396.

10. Gonzalez-Moles MA, Scully C. (2005) Vesiculo-erosive oral mucosal disease: management with topical corticosteroids: 1.Fundamental principles and specific agents available. J Dent Res. 2005; 84 (4): 294-301.

11. Sankar V, Hearnden V, Hull K, et al. (2011) Local drug delivery for oral mucosal diseases: challenges and opportunities. Oral Dis. 2011; 17 (Suppl 1):73-84.

12. Mark M. Schubert, Maria Elvira Pizzigatti Correa (2008) Oral Graft-Versus-Host Disease. The Dental Clinics of North America 52(2008) 79-109. doi:10.1016/j.cden.2007.10.004

13. Rosenberg PS, Greene MH, Alter BP. (2003) Cancer incidence in persons with Fanconi anemia. Blood. 2003; 101(3): 822-826.

14. Mawardi H, Elad S, Correa ME, et al. (2011) Oral epithelial dysplasia and squamous cell carcinoma following allogeneic hematopoietic stem cell transplantation: clinical presentation and treatment outcomes. Bone Marrow Transplant. 2011; 46 (6): 884-891. 


\section{CASE REPORT}

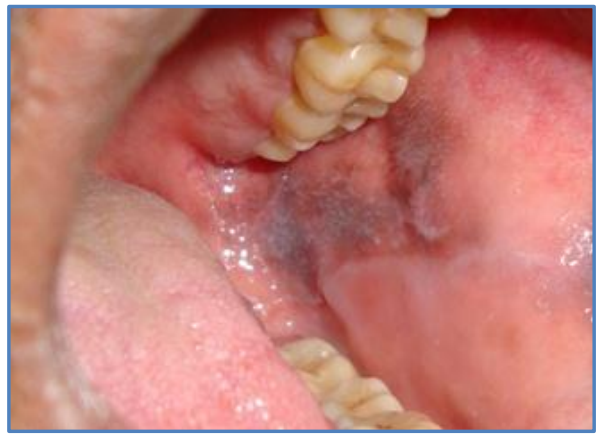

Fig. 1: Intra oral view showing a greyish discolouration in the left buccal mucosa with few white papules and mild diffuse erythematous area surrounding it.

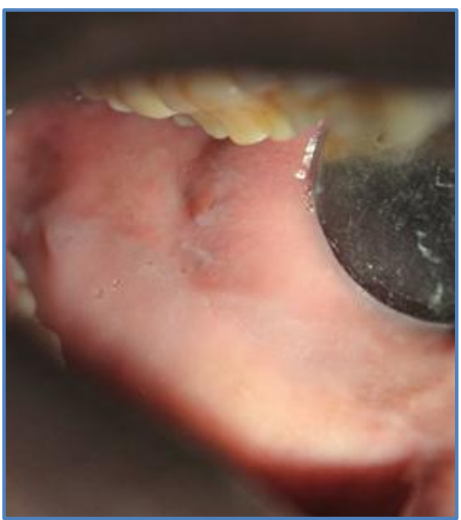

Fig. 3: Intra oral view showing left buccal mucosa after

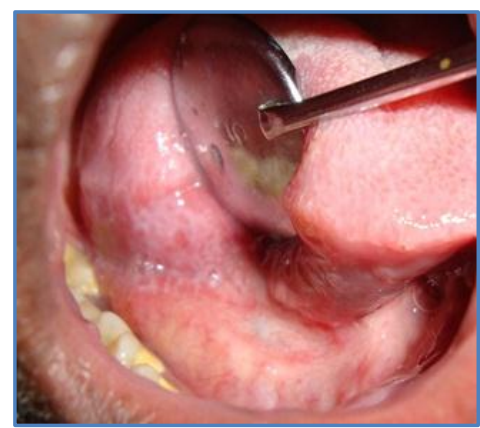

Fig. 2: Intraoral view showing a greyish white reticular pattern in the right lateral border of the tongue

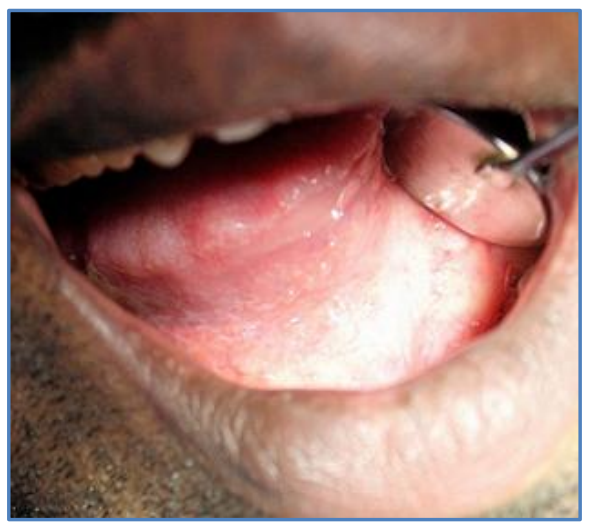

Fig. 4: Intra oral view showing right lateral border of the tongue after treatment

\section{AUTHORS:}

1. P. Redwin Dhas Manchil

2. Eugenia Sherubin

3. S. Karthiga Kannan

\section{PARTICULARS OF CONTRIBUTORS:}

1. Post Graduate Student, Department of Oral Medicine and Radiology, Sree Mookambika Institute of Dental Sciences, Kulasekharam, Kanyakumari District, Tamilnadu, India.

2. Reader, Department of Oral Medicine and Radiology, Sree Mookambika Institute of Dental Sciences, Kulasekharam, Kanyakumari District, Tamilnadu, India.

3. Professor, Department of Oral Medicine and Radiology, Mar Baselios Dental College, Kothamangalam, Ernakulam, Kerala.

\section{NAME ADDRESS EMAIL ID OF THE CORRESPONDING AUTHOR:}

Dr. Redwin Dhas Manchil, Post Graduate Student, Department of Oral Medicine and Radiology, Sree Mookambika Institute of Dental Sciences, Kulasekharam-629161, Kanyakumari District, Tamilnadu, India.

E-mail: redwinmanchil@gmail.com

Date of Submission: 07/05/2014. Date of Peer Review: 08/05/2014. Date of Acceptance: 26/05/2014. Date of Publishing: 09/06/2014. 